\title{
Prevalência de Candida spp. em amostras de secreção vaginal e sua relação com fatores associados à vulvovaginite
}

\author{
Prevalence of Candida spp. from samples of vaginal secretion and its \\ relationship to factors associated with vulvovaginitis
}

Marcia Barros Alves ${ }^{1}$; Ícaro Morais de Oliveira Silva ${ }^{1}$;Camilla Itapary dos Santos ${ }^{1}$, Yasmine Ramos França ${ }^{1}$; Suzane Kate Rocha Oliveira'; Sílvio Gomes Monteiro²; Cristina de Andrade Monteiro ${ }^{2}$

\begin{abstract}
Resumo: Objetivos: Determinar a prevalência de espécies de Candida provenientes de secreção vaginal verificando os principais fatores associados às infecções por Candida. Material e Métodos: Amostras foram coletadas de secreção vaginal por meio de esfregaço e isoladas em meio Sabouraud. Dados de anamnese preencheram um questionário. As leveduras encontradas foram identificadas pelo sistema de VITEK-2 e presença/ausência de Candida foi associada aos fatores relacionados a infecção pela levedura. O projeto foi aprovado pelo Comitê de Ética da Universidade Ceuma. Resultados: De um total de 144 pacientes, $62,5 \%$ apresentaram cultura positiva para Candida e destas a maior prevalência foi de C. parapsilosis (43\%). Entre os sinais e sintomas relatados e sugestivos para vulvovaginite por Candida , o corrimento foi o mais frequente ocorrendo em $24 \%$ das pacientes $(p<0,05)$.Dentre as pacientes positivas para presença de Candida, 59\% apresentavam ciclo menstrual regular e $19 \%$ faziam uso de anticoncepcional $(p<0,05)$. Quanto aos hábitos de vida, as variáveis mais prevalentes foram "um parceiro", "duas ou mais relações sexuais semanais", "uso de roupa de lycra" e "uso do protetor diário". Conclusões: Um percentual elevado de pacientes foi positivo para presença de Candida. Oito espécies diferentes do gênero foram identificadas, sendo a mais prevalente $C$. parapsilosis.
\end{abstract}

Palavras-chave: Vulvovaginite. Prevalência.Candida.Infecções.C.parapsilosis

Abstrac: Objectives: To determine the prevalence of Candida species from vaginal checking the main factors associated with Candida infections. Methods: Samples were collected vaginal swab through and isolation in Sabouraud anamnesis data and completed a questionnaire. Found yeasts were identified by two - VITEK system. The project was approved by the Ethics Committee of the Universidade Ceuma. Results: of 144 patients, $62.5 \%$ had positive culture for Candida and of these the greatest prevalence was C. parapsilosis (43\%). Of patients with signs and symptoms suggestive of Candida vulvovaginitis, $24 \%$ had discharge $(p<0.05)$. Among the patients positive for the presence of Candida, $59 \%$ have regular menstrual cycle and $19 \%$ use contraception $(p<0.05)$. As for lifestyle variables were more prevalent "a partner ", "two or more weekly sex ", " use of lycra clothing" and "protector use daily". Conclusions: A high percentage of patients analyzed was positive for the presence of Candida. Eight different species of the genus were identified, the most prevalent $C$. parapsilosis.

Keywords: Vulvovaginitis. Prevalence. Candida, infections. C. parapsilosis.

1 - Discente do Curso de Enfermagem - UNICEUMA

2 - Docente Permanente do Programa de Mestrado em Biologia Parasitária da Universidade CEUMA - Campus Renascença. Universidade CEUMA, Maranhão. 


\section{Introdução}

Vaginite é o problema
ginecológico mais comum entre
mulheres adultas e entre as
principais causas está Candida
spp $^{1}$. Candidíase vulvovaginal
(VVC) acomete grande parte das mulheres adultas pelo menos uma vez na vida. A candidíase vaginal recorrente (RVVC) é definida como sendo a que ocorre em quatro ou mais episódios sintomáticos de VVC em um ano ${ }^{2}$.

A maioria dos casos de candidíase vaginal é causada por Candida albicans, porém as espécies não-albicans foram também associadas com formas recorrentes ou crônicas da doença.

Mulheres com VVC
apresentam uma série de
manifestações desde a colonização assintomática até a infecção sintomática aguda severa. A maioria das mulheres assume, erroneamente, que todo e qualquer prurido genital é causado invariavelmente por uma candidíase, o que não é verdade, já que outros agentes podem determinar fluxos genitais. Além disso, outro problema consiste no fato de que a simples identificação da microbiota não significa necessariamente doença, daí a importância do melhor entendimento dos processos fisiopatológicos de VVC, assim como de dados epidemiológicos e fatores de risco, os quais permanecem inadequadamente estudados ${ }^{2}$.

Assim, esta pesquisa teve por objetivo isolar e determinar a prevalência de Candida spp. em secreção vaginal de mulheres atendidas no Hospital da Mulher (Programa Saúde da Mulher) e também verificar associações entre a presença de Candida e fatores relacionados à ocorrência de VVC.

\section{Material e Métodos}

Foi realizado um estudo transversal de caráter experimental com abordagem quantitativa durante o período de Outubro de 2010 a Maio de 2011, incluindo mulheres da comunidade do Anjo da Guarda, de regiões próximas e até de outras cidades do Estado do Maranhão, atendidas no Hospital da Mulher de São Luís para realização dos testes preventivos de câncer de colo uterino (colpocitologia papanicolau) através da procura espontânea para consulta.

O estudo foi aprovado pelo Comitê de Ética da Universidade Ceuma, Processo do Comitê de Ética em Pesquisa (CEP/UNICEUMA-267/10) e todas as pacientes que participaram da pesquisa assinaram o Termo de Consentimento Livre e Esclarecido. O critério de exclusão estabelecido foi a não participação de mulheres que tinham utilizado medicação antifúngica (oral/vaginal) nos últimos 30 dias.

As pacientes responderam a um questionário contendo perguntas sobre a presença de fatores de riscos associados a infecções do trato vaginal por Candida. As respostas dadas pelas pacientes ao questionário foram associadas à presença/ausência de Candida nas culturas.

Os dados foram avaliados pelo programa IBM SPSS Statistics 20 (2011).A avaliação estatística consistiu em apresentação dos resultados em números absolutos, 
percentuais e médias. Na análise univariada foram utilizadas tabelas de distribuição de frequências simples. A associação entre as variáveis "idade", "grau de instrução", "cor da pele", "estado civil", "atividade desenvolvida", "fatores de risco", "hábitos de vida", "manifestações clínicas" com cultura positiva para Candida spp. foi verificada por meio do teste Quiquadrado $\left(\chi^{2}\right)$ de independência. $O$ resultado foi considerado significativo quando $p<0,05$.

Para a cultura microbiológica foram retiradas amostras de 10 pacientes semanais atendidas durante os nove primeiros meses de pesquisa, independente dos sinais clínicos apresentados pelas mesmas.

A coleta foi feita por meio de esfregaço vaginal com a utilização de uma haste com algodão (swab) durante a colpocitologia (Papanicolau), retirando-se amostra cervicovaginal (colo uterino, fórnice posterior da vagina e paredes vaginais) e enviando este material em tubo estéril contendo solução salina $(0,85 \%)$ para o Laboratório de Micologia da Universidade Ceuma, onde as amostras foram semeadas em meio de cultura ágar Sabouraud, acrescido de cloranfenicol e incubadas a temperatura de $37^{\circ} \mathrm{C}$ durante 24 horas As amostras negativas foram mantidas por mais
48 horas em estufa para se confirmar a negatividade das culturas.

As colônias isoladas foram identificadas em meio CHRO Magar Candida-Agar Hicrome Candida (Himedia) e pelo sistema automatizado VITEK-2 (bioMériex, France) e após a identificação os isolados foram mantidos em meio Brain Heart Infusion (BHI) suplementado com $15 \%$ glicerol a $20^{\circ} \mathrm{C}^{3}$.

\section{Resultados}

Cento e quarenta e quatro (144) pacientes foram atendidas no serviço de Ginecologia do Hospital da Mulher em São Luís do Maranhão, no período de outubro de 2010 a maio de 2011.Destas, 90 pacientes $(62,5 \%)$ tiverem culturas positivas para leveduras do gênero Candida (Figura 1).

Foram identificadas 8 espécies diferentes pertencentes ao gênero Candida. Dentre estas $C$. parapsilosis $(\mathrm{n}=39 ; 43,3 \%), \quad C$. glabrata $(n=15 ; 16,7 \%)$ e C.albicans $(n=14 ; 15,6 \%)$ foram as mais frequentes. As demais espécies, $C$. tropicalis, C. famata, C. krusei, $C$. sakee $C$. globosa apresentaram frequência isolada inferior a $10 \%$ dos casos (Figura 2). 
Figura 1- Distribuição das amostras com culturas positivas e negativas para presença de leveduras do gênero Candida.

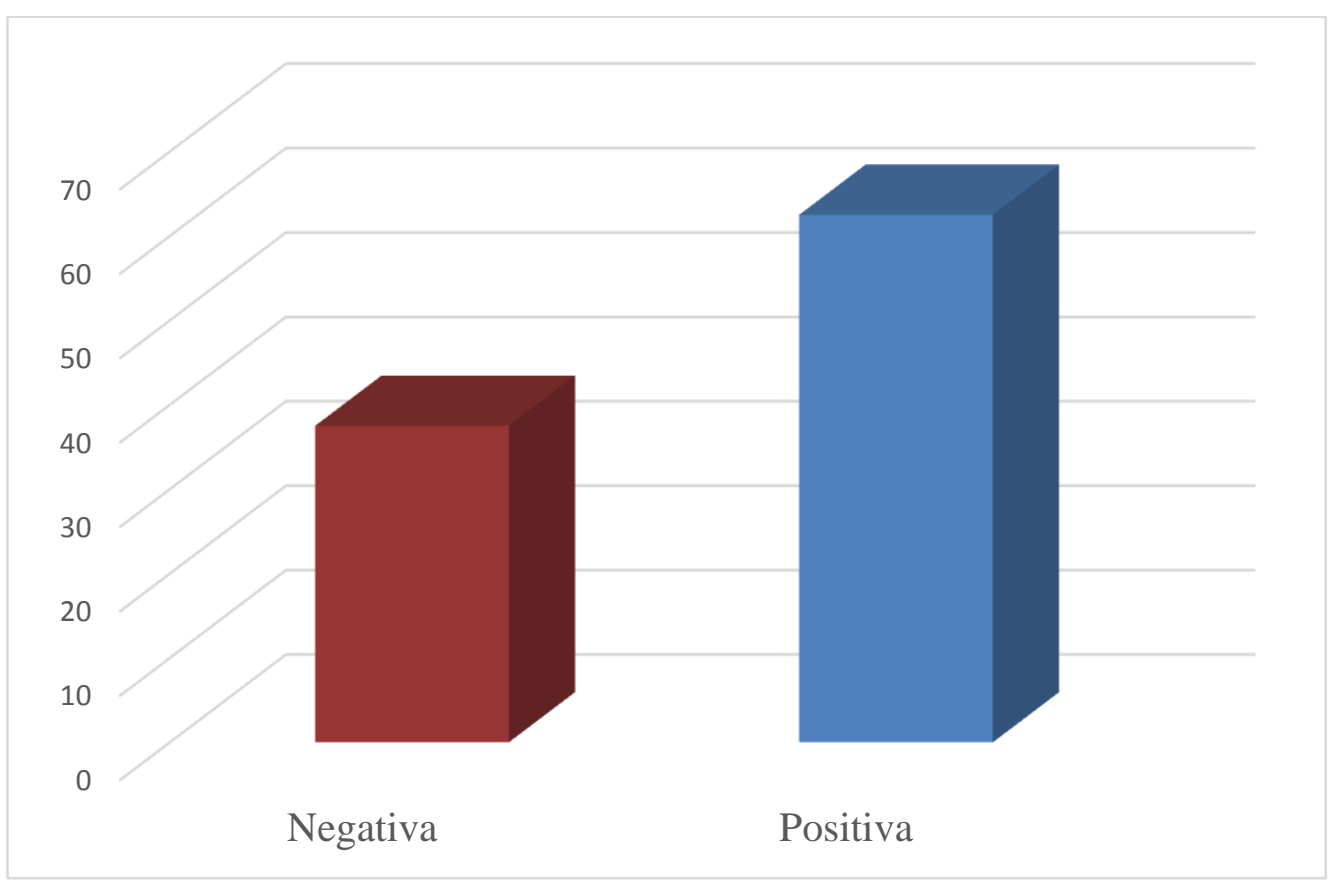

Figura 2- Frequência relativa das espécies de leveduras isoladas de secreção vaginal de pacientes atendidas no Hospital da Mulher em 2013. São Luís,MA

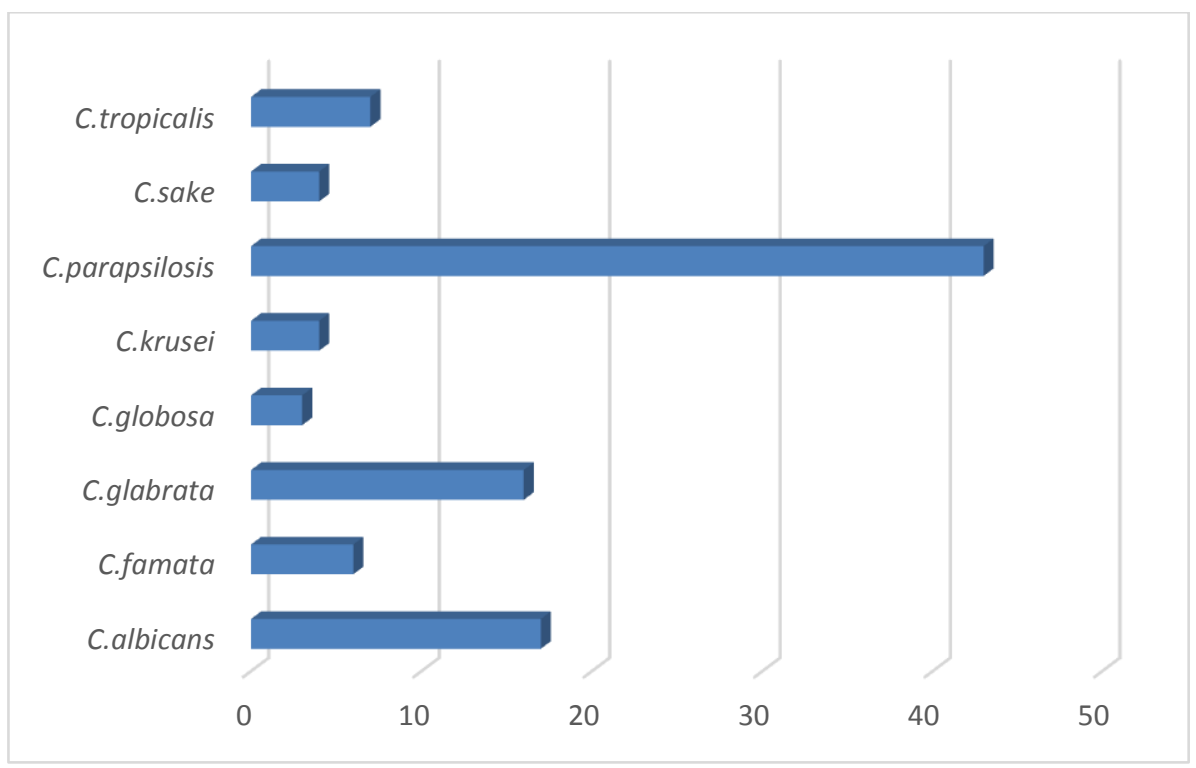


A tabela 1 apresenta a análise das pacientes que realizaram exame de cultura para leveduras do gênero Candida, em relação à faixa etária (15-64 anos).A presença de leveduras em secreções vaginais foi mais frequente em mulheres com idade entre 25 e 34 anos (35,55\%) e entre 35 e 44 anos (25,55\%), mas não houve associação $(p=0,07)$ entre a variável "idade" com culturas positivas e/ou negativas para Candida spp. Considerando-se o grau de instrução, a análise estatística mostra que houve associação entre a variável "grau de instrução"e a presença de leveduras do referido gênero $(p=0,01)$ e que as maiores frequências de presença da levedura foram observadas em mulheres que possuíam o ensino médio $(52,20 \%)$ e ensino fundamental(42,20\%).Alguns fatores considerados de risco para 0 desenvolvimento de VVC foram analisados, como mostrado na figura 3 e tabela 2 . Um caso de gravidez $(1,1 \%), 3$ casos de diabetes $(3,3 \%)$ e 3 casos $(3,3 \%)$ de D.S.T. (Doença Sexualmente Transmissível) foram constatados nas pacientes com culturas positivas. Embora não tenham sido registrados estes fatores nas pacientes com cultura negativa, estes achados não foram considerados estatisticamente significantes. Quanto aos outros fatores predisponentes para VVC, os resultados demonstraram maiores percentuais de mulheres com cultura positiva para Candida spp. $(\mathrm{p}<0,05)$ que relataram ter as variáveis: "ciclo menstrual regular" $(70 \%)$, "vulvovaginites prévias" $(80 \%)$ e "uso de anticoncepcional" $(20 \%)$.

Figura 3- Frequência de fatores predisponentes ao desenvolvimento de Vulvovaginites por Candida (VVC) relatados por pacientes atendidas no Hospital da Mulher em 2013, São Luís-MA.

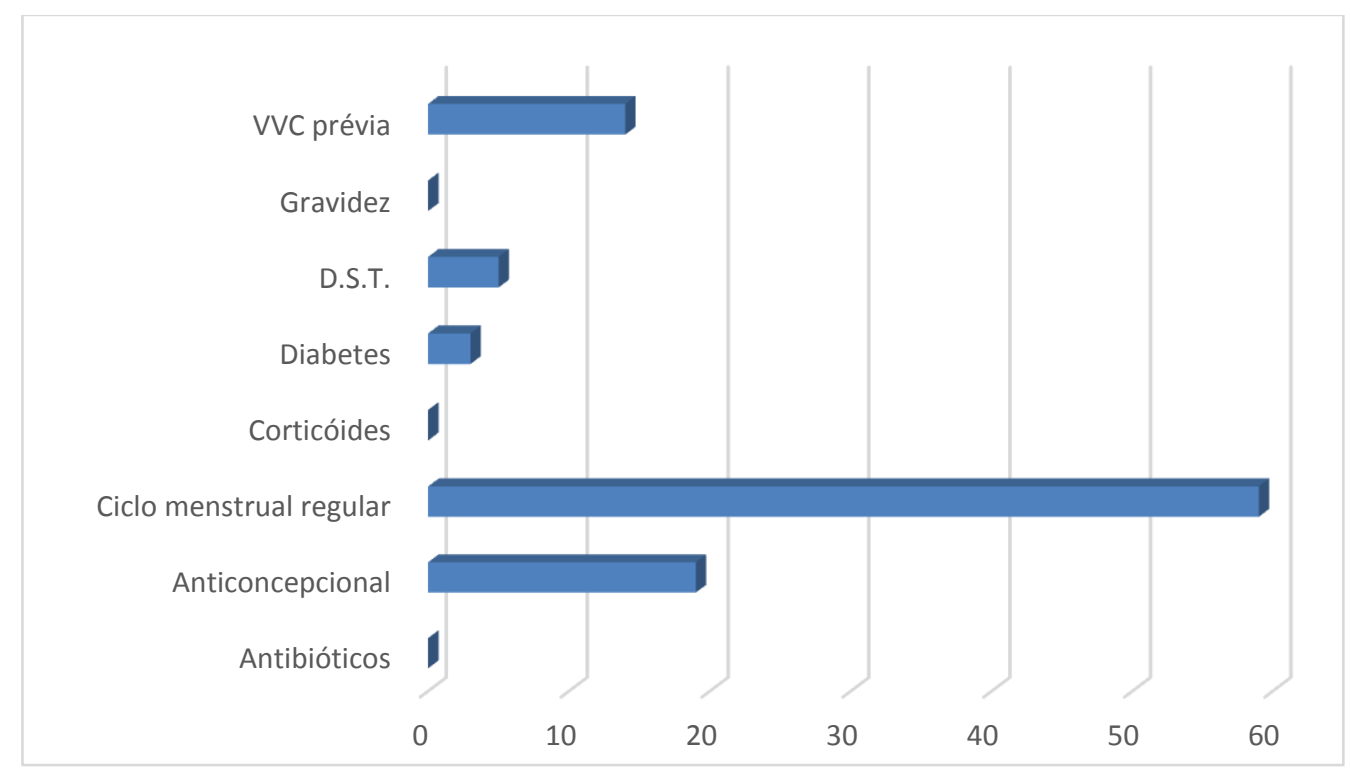


No que se refere aos hábitos de vida, as pacientes com cultura positiva referiram uso de roupa de lycra em $52,2 \%$ dos casos e de protetor diário em 21,1\%. Estes achados nas pacientes com cultura negativa atingiram valores de $14,8 \%$ e $3,7 \%$ respectivamente, o que foi considerado como estatisticamente significante $(p<0,05)$. Apenas 3 pacientes com cultura positiva $(3,3 \%)$ referiram uso de duchas higiênicas. A maioria das pacientes com cultura positiva possuía apenas 1 parceiro $(84,4 \%)$ e mantinham em média duas ou mais relações por semana $(64,4 \%)$, mas não houve associação entre estes fatores e presença de Candida nas culturas (p>0,05) (Tabela 2).

Os sinais e sintomas clínicos registrados pelas pacientes com maior frequência foram prurido, corrimento e dispaurenia. Houve correlação entre apresentação de corrimento relatadopelas pacientes e presença de Candida spp. na cultura $(p=0,009)$. Não houve correlação estatística entre a frequência dos sinais e sintomas relatados e as espécies de leveduras identificadas (Tabela 3).

Analisando a distribuição de pacientes assintomáticas com cultura positiva, pode-se observar que dentre as espécies de Candida, - percentual mostrou-se mais elevado em $C$. parapsilosis com 21 casos (44,6 \%) (Tabela 4).Com o objetivo de avaliar se houve alguma manifestação clínica patognomônica por espécie, associou-se os sinais e sintomas relatados pelas pacientes com as espécies das culturas positivas (Tabela 4). Observa-se que estas tem maior probabilidade de serem infectadas por Candida parapsilosis do que por outras espécies de Candida encontradas neste estudo $(p=0,0002)$. As pacientes com candidíase podem cursar com um quadro assintomático ou apresentar corrimento durante o quadro clínico. Os outros sintomas não foram considerados característicos de candidíase $(p=0,01)$.

\section{Discussão}

A vulvovaginite por levedura do gênero Candida (VVC)é uma patologia comum que corresponde ao segundo lugar entre as causas das vulvovaginites ${ }^{4}$.

Nesta pesquisa que incluiu 144 mulheres que procuraram voluntariamente 0 programa preventivo de câncer de colo uterino, a prevalência de pacientes com cultura positiva para leveduras do gênero Candida foi de $62,5 \%$. Gunther et al. ${ }^{4}$, em estudos comparativos entre mulheres com diabetes melitus e mulheres sem diabetes com sinais e sintomas de VVC, encontraram dentro do grupo de mulheres sem diabetes um total de $11,8 \%$, tendo uma taxa de mulheres com Candida spp. abaixo do encontrado neste estudo. Contudo, Rodrigues et al. $^{5}$ verificaram uma frequência de $79,9 \%$ de positividade de Candida spp. em cultura de secreção vaginal, valores estes mais próximos aos encontrados nesta pesquisa. Estas diferenças podem ocorrer em virtude da população estudada ter efetuado procura espontânea em um serviço especializado de um hospital de referência para a mulher ou pelo fato de que algumas pacientes desta população possam ter recebido anteriormente um diagnóstico incorreto e, 
consequentemente, um tratamento inadequado, uma vez que 0 diagnóstico correto de VVC requer cuidados especiais com a realização de exames de cultura e a utilização de meios cromogênicos para diferenciação das espécies de Candida que devem ser utilizados rotineiramente objetivando evitar 0 tratamento inadequado desta patologia 5 .

Dentre as culturas positivas, oito espécies diferentes de Candida foram identificadas e as mais prevalentes foram $C$. parapsilosis (43,3\%), C. glabrata (16,7\%) e C. albicans (15,6\%). C. parapsilosis foi a principal espécie encontrada neste estudo e este resultado em relação às demais espécies foi considerado significante $(p<0,05)$. Estes resultados são relevantes porque poderiam indicar uma tendência de mudança na etiologia de uma suposta candidíase, em mulheres que provavelmente tenham sido consideradas estar com VVC, após décadas de domínio de Candida albicans. Entretanto, $C$. parapsilosis não é considerada patógeno frequente em VVC. Guntheret al. ${ }^{4}$, em seu grupo controle de mulheres com Candida spp. e sem diabetes detectaram elevada prevalência de $C$. albicans $(54,4 \%)$ em detrimento das demais espécies de Candida não $C$. albicans $(45,6 \%)$, assim como no estudo de Rodrigues et al. ${ }^{6}$, no qual a $C$. albicans $(98,1 \%)$ também foi a mais prevalente.A frequência de $C$. parapsilosis encontrada por Adrioli et al. ${ }^{7}$, que trabalharam com amostras de secreção vaginal, foi de $5,3 \%$,o que também difere dos resultados obtidos no presente trabalho. Mas, Kumariet al. ${ }^{8}$, corroborando os resultados obtidos aqui, detectaram maior prevalência de C. parapsilosis $(45,07 \%)$ em relação a $C$. albicans $(32,39 \%)$ e $C$. glabrata $(22,53 \%)$ em mulheres com sintomas de VVC.

O maior registro de espécies de Candida não $C$. albicans pode ser decorrente de uma maior utilização de exames de culturas e testes de identificação ${ }^{5}$. Em estudos epidemiológicos deve-se considerar também a variação de acordo com a localização geográfica e o tratamento usado. Segundo Sobel ${ }^{9}$, um provável aumento das vaginites causadas por $C$. parapsilosis, $C$. tropicalis e C. krusei, apesar de consideradas pouco frequentes, pode estar relacionado a tratamento em dose única ou a uma dosagem baixa da droga no tratamento e manutenção.Nos Estados Unidos houve um aumento nos casos de VVC ocasionadas por espécies de Candida não $C$. albicans devido ao uso generalizado de antifúngicos orais $^{9}$. Além disso, a automedicação por parte da população no que diz respeito a tratamento popular por fitoterápicos ou quaisquer outras substâncias poderia facilitar a multiplicação de espécies de Candida antes não prevalentes ou de espécies resistentes a estas substâncias. Estes fatos também poderiam contribuir para os resultados de prevalência obtidos.

A faixa etária da população participante neste estudo variou entre 15 e 64 anos e a presença de Candida nas culturas foi mais prevalente em mulheres entre $25 \mathrm{e}$ 34 anos (35,55\%). Embora não tenha sido registrada diferença significante entre as culturas positivas e negativas para Candida em relação à variável "idade" $(p=0,07)$, resultado semelhante a este foi encontrado por Andrioliet al. ${ }^{7}$, que registraram uma maior 
prevalência do gênero entre as idades de 14 a 46 anos.

Foi observado que a presença de Candida foi mais frequente em mulheres que haviam cursado até 0 ensino médio $(52,2 \%)$, resultado considerado estatisticamente significante $(p=0,01)$ seguindo-se de pacientes que possuíam ensino fundamental $(42,2 \%)$. Um dos fatores que poderia estar associado à positividade para Candida em pacientes com grau de instrução menor seria a condição inadequada de higiene.

Com relação aos fatores comumente considerados como predisponentes ao desenvolvimento de VVC avaliados no questionário, não foi encontrada associação significante entre "gravidez" e presença de Candida ( $p>0,05)$, e também entre as variáveis "diabetes" e "doenças sexualmente transmissíveis" com presença de leveduras. Isto se deve ao fato de apenas uma paciente $(1,1 \%)$ ter referido estado gestacional e somente $3,3 \%$ apresentarem as condições "diabetes" e ter "doenças sexualmente transmissíveis". Dias et al. ${ }^{10}$ observaram uma prevalência de VVC em mulheres grávidas de $44,8 \%$ enquanto que as não gestantes apresentaram prevalência de $34,5 \%$.Sabe-se que a VVC pode ocorrer na gestação, sendo mais frequente na $28^{\mathrm{a}}$ semana, pois nesta fase ocorre um aumento do nível de glicogênio no epitélio vaginal por estímulo dos níveis de estrógeno gestacional ${ }^{11}$, ocasionando maior acidez, o que facilitaria 0 crescimento de leveduras.O mesmo pode ocorrer com pacientes portadoras de diabetes descompensado que possuem maior predisposição para o desenvolvimento de VVC, bem como para aquelas que fazem uso de anticoncepcionais em doses elevadas.

Alguns destes relatos corroboram os resultados deste estudo que mostraram 11 pacientes $(12,2 \%)$ com cultura positiva para Candida fazendo uso de anticoncepcional oral, dado este considerado estatisticamente significante $(p=0,01)$. Não houve registro deste uso nas pacientes com culturas negativas

Esta pesquisa registrou que $18,8 \%$ das pacientes com presença de Candida nas culturas relataram ter tido episódios anteriores de VVC $(p<0,05)$ e $70 \%$ referiram ter ciclo regular, dado considerado elevado e estatisticamente significante $(p<0,001)$. A literatura registra percentuais elevados de pacientes com candidíase vaginal que relataram ter tido VVC prévia e apresentaram ciclo menstrual regular como os estudos de Corrêa et al. $^{13}$. A acidez que poderia favorecer a instalação da infecção fúngica ocorreria devido aos picos hormonais que existem neste tipo de ciclo, como o que é evidenciado na fase lútea do ciclo menstrual ${ }^{7}$.

Quanto aos hábitos de vida, o mais prevalente manifestado pelas pacientes analisadas foi o "manter relações com apenas um parceiro" com frequência de $84,4 \%$. Entretanto, este dado não mostrou relação com presença de Candida nas culturas $(p>0,05)$ apesar de a atividade sexual poder atuar como uma forma de disseminação de Candida ${ }^{4}$. Não foi registrada também relação entre presença da levedura e uso de duchas higiênicas.

Associação significante com cultura positiva para Candida foi 
encontrada para a variável "uso de roupas de lycra" $(p<0,001)$ o que está de acordo com os resultados obtidos por Andrioliet al. ${ }^{7}$ e para "uso de protetor diário" $(p<0,05)$; de acordo com Patel et al. ${ }^{10}$ a pouca aeração nos órgãos genitais provocada pelo uso de roupas justas e/ou sintéticas resulta no aumento da umidade no local o que predispõe a VVC.

Rodrigues et al. ${ }^{6}$ observaram como sendo fatores predisponentes mais comuns associados com a candidíase a gravidez, uso de contraceptivos, atividade sexual e higiene, corroborando os dados obtidos no presente trabalho ${ }^{14}$.

A porcentagem de pacientes que relataram ter sinais e sintomas relacionados a VVC não foi maior que as consideradas assintomáticas e uma relação entre as sintomáticas e presença de Candida não foi estabelecida.Corrêa et al. ${ }^{12}$ referem que VVC ocasionadas por espécies de Candida não $C$. albicans podem ocorrer em mulheres assintomáticas em até $44 \%$ dos casos.Os blastóporos poderiam se alojar em camadas mais profundas, ficando protegidos da ação de antifúngico e posteriormente após um período, que poderia durar semanas, iriam novamente para a luz vaginal reiniciando 0 processo com sintomas.

\section{Pode-se}

associar manifestação clínica de corrimento $(p<0,05)$ com cultura positiva para Candida o que é corroborado pelos relatos de outros autores ${ }^{9,11,16}$. Outros sinais e sintomas mais frequentemente relatados foram prurido, ardor vaginal e dispaurenia. Algumas manifestações clínicas costumam ser encontradas em pacientes com VVC, como prurido e corrimento $^{12}$. Entretanto, não foi encontrada associação entre as espécies de Candida identificadas e os sinais e sintomas de vulvovaginites relatados $(p>0,05)$.

Estes resultados são relevantes, pois mostram associação entre pacientes com Candida e com algumas variáveis consideradas fatores predisponentes para VVC, como "vulvovaginites prévias", "uso de anticoncepcionais orais" e "ciclo menstrual regular"; e com hábitos de vida, como "uso de roupa de lycra" e "uso de protetor diário". Isto indicaria uma maior probabilidade de desenvolvimento de VVC nestas pacientes e evidencia a necessidade de um exame clínico para diagnóstico e confirmação da infecção nas mesmas.

O diagnóstico preciso da espécie de Candida em mulheres que apresentam sinais e sintomas associados à VVC deve ser feito associando-se o exame direto das secreções vaginais e a cultura objetivando evitar 0 tratamento inadequado de uma provável patologia o que pode culminar com resistência a drogas e com recidivas. A identificação de Candida em mulheres assintomáticas não requer tratamento, mas uma orientação quanto aos fatores predisponentes.

\section{Conclusões}

Pode-se concluir por meio desta pesquisa que:

- Um percentual elevado de pacientes analisadas(62,5\%) foi positivo para presença de leveduras do gênero Candida em amostras de secreção vaginal;

- Dentre as culturas positivas, oito espécies diferentes de Candida 
foram identificadas, sendo a mais prevalente $C$. parapsilosis (43,3\%);

- As pacientes com Candida apresentam associação com algumas variáveis de padrão demográfico e social. Como "grau de instrução"; e com hábitos de vida, como "uso de roupa de lycra" e "uso de protetor diário".;

- O corrimento pode ser considerado um sinal para ocorrência de Candida em amostras de secreção vaginal já que a relação com esta variável foi estatisticamente significante.

\section{Referências}

1. Nouraei $S$, Akbari $S$, Jorjani $M$, Majd $\mathrm{H}$, Afrakhteh $\mathrm{M}$, Ghafoorian A. Comparison between fluconazole with oral protexin combination and fluconazole in the treatment of vulvovaginal candidiasis. Isrn obstetrics and gynecology.2012.

2. Heng L, Hen Y, Tan, T. Treatment of recurrent vulvo-vaginal candidiasis with sustained-release butoconazole pessary. Singapore medical journal. 2012; 53: 269271.

3. Sambrook J, Frstch E, Maniatis T. Molecular cloning : a laboratory manual; cold spring harbor: new york. 2002.

4. Gunther L, Martins H, Gimenes F, Abreu A, Consolaro $M$, Svidzinski T. Prevalence of candida albicans and non-albicans isolates from vaginal secretions: comparative evaluation of colonization, vaginal candidiasis and recurrent vaginal candidiasis in diabetic and nondiabetic women. São paulo medical journal. 2014; 132.
5. Mahmoudabadi A, Najafyan M, Moghimipour E, Alwanian M, Seifi $Z$. Lamisil versus clotrimazole in the treatment of vulvovaginal candidiasis. Iran journal microbiology. 2013; 86-90.

6. Rodrigues M, Gonçalvez A, Alvim M, Filho D, Zimmermmann J, Silva V. Association between vaginal secretion culture, sociodemographic characteristics and clinical manifestations of patients with vulvovaginal candidiasis]. Revista brasileira de ginecologia e obstetrícia.2013; 35.

7. Andriolli J. Frequency of yeast in vaginal fluido women with and without clinical suspicion of vulvoginal candidiasis. Revista brasileira de ginecologia e obstetrícia. 2009; 31: 300-304.

8. Kumari V, Banerjee $\mathrm{T}$, Kumar $\mathrm{P}$, Pandey S, Tilar R. Emergence of non-albicans candida among candidal vulvovaginitis cases andstudy of their potential virulence factors, from a tertiary care center, north india. Indian journal of pathology \& microbiology. 2013; 56: 144-147.

9. Sobel J. Vulvovaginal candidosis. Lancet. 2007; 369, 1961-1971.

10. Patel D, Gillespie B,Sobel J, Leaman D, Nyirjesy P, Weitz M. Risk factors for recurrent vulvovaginal candidiasis in women receiving maintenance antifungal therapy: results of a prospective cohort study. Am j obstet gynecol. 2005;190(3):644-53.

11. Dias L, Melhem M, Szeszs M, Filho J, Hahn R. Vulvovaginal candidiasis in mato grosso, brazil: pregnancy status, causative species and drugs tests. Braziliam journal microbiology.2011; 13001307. 
12. Zimmerman J. Validade do diagnóstico clínico de candidíase vulvovaginal. Hu revista. 2009;35: 11-18.

13. Correa P. Phenotypic characterization of yeasts isolated from the vaginal mucosa of adult women. Revista brasileira de ginecologia e obstetrícia. 2009; 31:177-181.

14. Soong D, Einarson A. Vaginal yeast infections during pregnancy. Canadian family physician. 2009; 55: 255-256. 